\title{
La armonización territorial: su incorporación en la planificación y gestión administrativa mediante la gestión del riesgo*
}

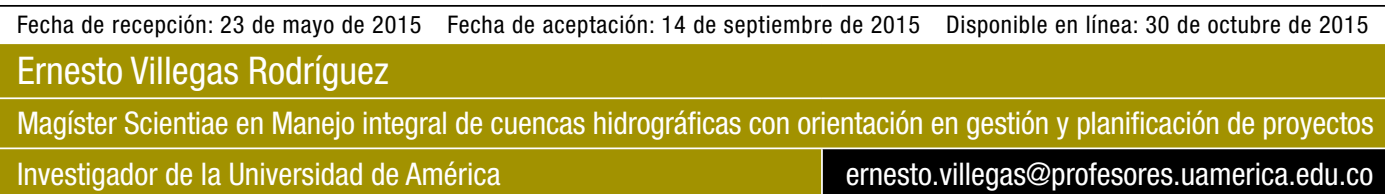

Resumen A lo largo del artículo emergen varios elementos con los cuales se puede establecer la incidencia de las políticas públicas en las tomas de decisión en el territorio, sobre todo en la utilización de tres instrumentos de planificación y de gestión territorial como el Plan de Desarrollo (PD), el Plan de Ordenamiento Territorial (POT) y los Planes de Manejo Integral de Cuencas Hidrográficas (Pomca), estructurados por medio de las Unidades de Planificación y de Gestión Territorial (UPGT). Estos permiten articular el desarrollo de programas y proyectos en un territorio asociado en municipios y departamentos, con el fin de invertir y localizar infraestructuras que reduzcan la vulnerabilidad y amenaza en áreas que han sido objeto de deslizamientos e inundaciones e intervenidas por el Gobierno nacional mediante la Ley 1523 de 2012 de Gestión del Riesgo y complementadas por otras normas que fortalecen la dimensión ambiental, social, económica y administrativa que coadyuvan a un ordenamiento territorial más integral. La participación de actores y sectores públicos y privados y la sociedad civil son coordinados con la puesta en marcha de los Comités de Integración Territorial (CIT), que jalonan los procesos administrativos alrededor del riesgo y de los desastres naturales asociados, en muchos casos, con las actividades humanas.

Palabras clave cuenca hidrográfica; Sistema de Información Nacional Ambiental; instrumentos de gestión y planificación territorial; Sistema Nacional para la Gestión del Riesgo de Desastres; Programa Agua para la Prosperidad; unidades de gestión territorial

\footnotetext{
Artículo resultado de investigación científica y tecnológica, titulada "Proponer un modelo de asociación territorial a escala urbano-rural, que integre estrategias de diseño urbano arquitectónico de equipamiento y espacio público, para generar núcleos de unidades asociativas socioeconómicas y ambientales de gestión, como instrumento operativo en los POT y PDM", patrocinada por la Facultad de Arquitectura de la Fundación Universidad de América. Código de registro: TH1.1.01.02.14 Feb 2014. Fecha de inicio: febrero 1 de 2014.
}

Cómo citar éste artículo: Villegas, E. (2015). La armonización territorial: su incorporación en la planificación y gestión administrativa mediante la gestión del riesgo. Cuadernos de Vivienda y Urbanismo, 8(16), 148-165. http://dxdoi.org/1011144/Javeriana.cvu8-16.atip 


\section{Territorial Harmonization: Its Additions in Planning and}

Administrative Management through Risk Management

Abstract Throughout the article several elements which can set the impact of public policies in territory decision making emerge. Especially the use of three instruments of territorial planning and management as the Development Plan (Plan de Desarrollo, PD), the Land Use Plan (Plan de Ordenamiento Territorial, POT) and the Plans for Comprehensive Watershed Management (Planes de Manejo Integral de Cuencas Hidrográficas, Pomca), structured through the Units of Planning and Land Management (Unidades de Planificación y de Gestión Territorial, UPGT). These allow the development of programs and projects in a territory associated in municipalities and departments in order to invest and locate infrastructures that reduce vulnerability and threat in areas that have been subject to landslides and floods and taken over by the National Government through the Law 1523 of 2012 on Risk Management and supplemented by other regulations that strengthen the environmental, social, economic and administrative dimension that contribute to a more comprehensive land use. The participation of public and private actors and sectors as well as of civil society is coordinated with the implementation of the Committees for Territorial Integration (Comités de Integración Territorial, CIT), which connect the administrative processes around risk and associated natural disasters, in many cases, with human activities.

Keywords watershed; National Environmental Information System (Sistema de Información Nacional Ambiental); management and territorial planning tools; National System for Disaster Risk Management (Sistema Nacional para la Gestión del Riesgo de Desastres); Water for Prosperity Programme (Programa Agua para la Prosperidad); land management units

\section{A harmonização territorial: sua incorporação no planejamento e} gestão administrativa mediante a gestão do risco

Resumo Ao longo do artigo emergem vários elementos com os quais pode se estabelecer a incidência das políticas públicas nas tomadas de decisão no território, sobre todo na utilização de três instrumentos de planejamento e gestáo territorial como o Plano de Desenvolvimento (PD), o Plano Diretor (POT, pela sua sigla em espanhol) e os Planos de Maneio Integral de Bacias Hidrográficas (Pomca, pela sua sigla em espanhol), estruturados por meio das Unidades de Planejamento e Gestão Territorial (UPGT). Estes permitem articular o desenvolvimento de programas e projetos em um território associado em municípios e departamentos ${ }^{1}$, a fim de investir e localizar infraestruturas que reduzam a vulnerabilidade e ameaça em áreas que já foram objeto de escorregamentos e inundaçóes e intervindas pelo Governo nacional através da Lei 1523 de 2012 de Gestão do Risco e complementadas com outras normas avigorando a dimensão ambiental, social, económica e administrativa que coadjuvam para um ordenamento territorial mais abrangente. A participação de atores e setores públicos e privados e a sociedade civil é coordenada com a implementação dos Comités de Integração Territorial (CIT), que puxam os processos administrativos em torno do risco e os desastres naturais associados, em muito caso, com atividades humanas.

Palavras chave bacia hidrográfica; Sistema de Informação Nacional Ambiental; instrumentos de gestão e planejamento territorial; Sistema Nacional para a Gestão do Risco de Desastres; Programa Agua para a Prosperidade; Unidades de Gestão Territorial 


\section{Antecedentes}

En Colombia, la gestión del riesgo es considerada la columna vertebral en términos de planificación, gestión y ordenamiento territorial en el corto, mediano y largo plazo, lo cual debe abordarse de acuerdo con su incidencia sobre el territorio mediante los Planes de Desarrollo (PD), los Planes de Ordenamiento Territorial (POT) y los Planes de Manejo Integral de Cuencas Hidrográficas (Pomca).

Respecto al documento de la Procuraduría General de la Nación para Asuntos Ambientales y Agrarios, se destaca el concepto del Ideam de julio del año 2007, que afirma que no se tienen políticas claras de ordenamiento y uso de los recursos hídricos y también evidencia que los grandes asentamientos humanos y los polos de desarrollo industrial, agrícola, pecuario e hidroenergético en el país se han presentado en regiones donde la oferta hídrica es menos favorable, lo que genera presiones sobre el recurso y problemas de disponibilidad de agua para algunos municipios y sus áreas urbanas durante períodos de condiciones climáticas extremas, como las épocas secas y de influencia del fenómeno cálido del Pacífico (El Niño). También existen grandes problemas referentes al tema de la infraestructura vial, localizada en áreas de alto riesgo por deslizamiento de tierra e inundación en época de lluvia, conocida como el fenómeno de La Niña.

Desde la visión de la Ley 388 de 1997, la dimensión ambiental hace referencia a los procesos sociales y políticos en la construcción del territorio, por medio de los cuales cada una de las Administraciones municipales busca controlar los procesos de ocupación y construcción de asentamientos humanos en armonía con el ambiente, en especial para algunos asentamientos que, por las condiciones sociales y económicas de su ocupación territorial, se han ubicado en zonas de alto riesgo; para ello, mediante el Plan de Desarrollo municipal se han tenido que destinar grandes recursos financieros para la prevención y atención de desastres.

Algunas inversiones están destinadas a la infraestructura social, que posibilita el desarrollo de actividades económicas, representadas por las obras relacionadas con vías de segundo y tercer orden, de competencia de los municipios y otras obras de infraestructura como puentes, sistemas de riego, suministro de agua potable, alcantarillado, viviendas, escuelas y hospitales, entre otras, que son instalaciones que son las que más han sufrido en eventos naturales, debido al grado de vulnerabilidad y amenaza frente al riesgo por deslizamiento o inundación. Parece paradójico, pero la mayoría de las cabeceras municipales en Colombia presentan estas características.

En términos de atención y prevención de desastres, el fortalecimiento institucional ha demostrado avances significativos, con gran pronunciamiento de normas, decretos y leyes que hacen compleja su articulación y coordinación entre las competencias de ley de municipios, departamentos y la Nación, al momento de tomar decisiones en el territorio, como lo comprobado 
en 2010-2011 por la incidencia del fenómeno de La Niña, cuando se registraron las más altas precipitaciones de los últimos sesenta ańos (Ideam, 2011) y que ocasionó daños en infraestructura vial, pérdida de asentamientos conocidos como cabeceras municipales, centros poblados anegados en su totalidad y pérdidas de acueductos y alcantarillados, en especial en la región Caribe. Hasta el momento no se han encontrado soluciones estratégicas, lo que ha llevado a la reestructuración de algunas normas, entre ellas, la Ley 99 de 1993, sobre las funciones de las Corporaciones Autónomas Regionales (CAR), a las que les corresponde el cumplimiento de la legislación ambiental.

Por lo anterior, el Gobierno central expidió el Decreto 4147 del 3 de noviembre de 2011, en el que se determina la creación de la Unidad $\mathrm{Na}$ cional para la Gestión del Riesgo y de Desastres. En su Artículo 3 describe su objetivo principal:

[...] dirigir la implementación de la Gestión del Riesgo de Desastres, atendiendo las políticas de desarrollo sostenible, y coordinación al funcionamiento y el desarrollo continuo del Sistema Nacional para la Prevención y Atención de Desastres (SNPAD), buscando mejorar la coordinación al interior del Sistema Nacional para la Prevención y Atención de Desastres.

En términos de complementariedad, en el año 2012 se expidió la Política Nacional de Gestión del Riesgo de Desastres, conocida como la Ley 1523, para fortalecer los procesos de desarrollo sostenible y de seguridad integral de la población, al considerar la gestión del riesgo como una dimensión del desarrollo y de la institucionalidad en el territorio. Referente a la gestión territorial, su trascendencia es la homogenización de toda la normatividad, con el fin de disminuir la duplicidad de esfuerzos entre las Administraciones o entidades públicas que, por la vasta información legal y normativa sobre el tema de riesgo las han hecho inoperantes.
Además, el tema del riesgo crea una articulación con los instrumentos de gestión y de planificación territorial como los Pomca, Planes de Desarrollo y Planes de Ordenamiento Territorial, que son las instancias que determinan las condiciones de localización de infraestructura e inversión de recursos públicos. Vale la pena resaltar que en la Ley 1523 de 2012 se asigna responsabilidad total al individuo por su localización.

Como se ha venido diciendo, son innumerables los decretos o el marco jurídico en temas relacionados con los desastres a partir de la erupción del Nevado del Ruiz, el 13 de noviembre de 1985, conocida como la avalancha que sepultó a la ciudad de Armero; la ineficacia gubernamental por la pérdida de más de 30.000 vidas fue acogida como experiencia por los expertos en erupciones volcánicas del Gobierno de Filipinas para tomar decisiones sobre el desalojo de más de un millón de habitantes por la erupción del volcán Pinatubo en 1991.

Hoy en día se busca establecer la interrelación en la política pública colombiana en cuanto a la coordinación, complementariedad y subsidiaridad entre las distintas instituciones de las Administraciones territoriales como municipio, departamento y Nación en la atención del desastre. Se puede decir que las CAR serán las posibles coordinadoras en procesos de atención y prevención de desastres, con la aplicación de la Ley 1523 de 2012 de la Gestión del Riesgo, aunque también se espera una profunda reforma por normas presidenciales de la Ley General Ambiental de Colombia, Ley 99 de 1993.

Otra condición extrema de amenaza y vulnerabilidad territorial es la geografía colombiana, que condiciona el desarrollo y el crecimiento económico del país a la topografía y a la conectividad y competitividad regional. Por más pérdidas que hayamos registrado en los últimos tiempos y acciones puestas en marcha por Gobiernos 
mediante sus Planes de Desarrollo y Planes de Ordenamiento Territorial, no se ha logrado aprender de nuestro ambiente geohidrológico para llevar a cabo diseńos de planificación y de gestión territorial a largo plazo. Se sabe que los sistemas hídricos que abastecen a la población colombiana evidencian una alta vulnerabilidad y no garantizan una disponibilidad adecuada de agua, pues se nutren de fuentes pequeñas, arroyos, quebradas y riachuelos con bajas condiciones de regulación, disponibilidad y alta vulnerabilidad a los deslizamientos, conocidas como microcuencas abastecedoras de acueductos municipales, como lo sucedido en la ciudad de Yopal (Casanare), entre otras ciudades.

A continuación se determinan tres sectores característicos importantes que deben tenerse en cuenta en el marco referencial, para proyectar la evaluación de la gestión del riesgo en la incorporación de los instrumentos de planificación y gestión territorial para el planeamiento del territorio: el sector oficial, el sector privado y la sociedad civil. Se busca la participación de cada uno de ellos en el conocimiento de normas que conlleven a la disminución de pérdidas tanto humanas como económicas y fortalecer cada vez más la articulación entre las instituciones del Estado con las del sector privado y la sociedad civil en los contextos territoriales.

\section{Desarrollo, amenaza, vulnerabilidad y riesgo}

En distintas Administraciones municipales, departamentales y nacionales se ha logrado identificar cambios presupuestales de inversión mediante los Planes de Desarrollo, a consecuencia de los eventos naturales sucedidos en sus propios períodos administrativos; tales recursos han tenido que direccionarse para la atención de terremotos, deslizamientos e inundaciones. Por lo tanto, en el manejo de las inversiones y de las políticas públicas, el Gobierno central ha solicitado a los alcaldes y gobernadores crear el mapa de riesgo de desastres naturales, con el fin de determinar y localizar las inversiones futuras.

Al sancionarse la Ley 1523 de abril 2012, de Gestión de Riesgo de Desastres, la Presidencia de la República garantizó un trabajo más organizado para enfrentar los riesgos naturales, ya que estos deben estar incorporados en los instrumentos de planificación (Pomca, POT, PD), armonizados de manera que alcaldes y gobernadores puedan identificar zonas más vulnerables a los desastres naturales.

Para una mejor articulación del Sistema de Gestión del Riesgo, la Ley funcionará bajo la coordinación de la Presidencia de la República y tendrá como objetivo central desarrollar, mantener y garantizar el proceso de conocimiento del riesgo y de manejo del mismo, para que el país esté mejor preparado a la hora de atender desastres naturales y calamidades y puedan contar con todo un sistema articulado para trabajar en la reducción del riesgo, según el presidente de la República, Juan Manuel Santos (20102014), al promover la Ley como la primera en su género enfocada a prevenir y no solo a atender calamidades naturales.

La experiencia de Colombia frente a sucesos naturales ocurridos en décadas pasadas ubica al país en uno de los primeros lugares en donde la pérdida de vidas humanas está asociada con los eventos de sismos, deslizamientos y avalanchas, entre otros (Ideam, 2011) y ha llevado a la promulgación de normas específicas en la prevención y atención de los desastres, entre ellas, la Ley 1523 de 2012, con base en la Guía para la gestión del riesgo municipal de 2010. Tras los eventos conocidos durante el fenómeno de La Nińa 20102011 se afectó un gran número de territorios 
municipales, por lo que se declaró el estado de calamidad nacional y se incorporó una nueva estructura interinstitucional para enfrentar eventos en el futuro.

Lozano Vila (2011) identifica la vulnerabilidad para Colombia como la variabilidad climática, debido a que el número de eventos registrados es el mayor del continente, con una alta y creciente incidencia de emergencias asociadas con el clima. Según la autora, facilitadora y negociadora para el Cambio Climático, Desarrollo Sostenible Rio+20 para Colombia, no hay que olvidar que el país cuenta con una matriz energética, que proviene en un $85 \%$ de hidroeléctricas, pero es altamente vulnerable en una situación de fenómeno como El Niño o La Niña.

En su diagnóstico ambiental, la doctora Lozano Vila (2011) aclara que la gestión del riesgo es la columna vertebral de la visión que debemos tener sobre el tema ambiental y se compagina con la reciente Ley 1454 de 2011, que dicta las normas orgánicas para la armonización en este tema. El pasado Plan Nacional de Desarrollo 2010-2014 también resalta que los índices de precipitaciones presentados durante el mes de noviembre de 2010 superaron los registros históricos, lo que aumentó los niveles de los ríos Cauca y Magdalena y sus afluentes. Este fenómeno ha sido el más fuerte registrado desde 1949 y los impactos generados por La Niña han dejado más de 2,27 millones de personas afectadas.

Como parte del proceso de articulación entre el Estado y los entes territoriales municipales, con esta experiencia se hace necesario fortalecer los instrumentos de planificación y de gestión territorial, por medio de cuatro factores:

a) Factores físicos. Ubicación y resistencia material de los bienes en relación con el evento amenazante. b) Factores ambientales. Corresponde a la manera como la comunidad "explota" los elementos de su entorno natural y debilita al medio y a los ecosistemas.

c) Factores económicos. Corresponden a la ausencia de recursos económicos (pobreza).

d) Factores sociales. Se trata de los aspectos políticos, organizacionales, institucionales, educativos y culturales del municipio.

En cuanto a los factores físico, ambiental, social y económico, la Guía metodológica de desarrollo municipal para los riesgos y desastres 2010 alude a la importancia de reconocer el pasado como historia en la ocurrencia de nuevos eventos que deben ser tenidos en cuenta en la localización de infraestructuras viales; la Guía hace mención a la gestión del riesgo desde la necesidad de orientar la gestión territorial en función del riesgo en el mismo momento en que inicien los procesos de ordenación y planificación territorial. Así, podríamos consolidar la gestión del riesgo como estrategia en la verdadera planificación territorial; sin embargo, la gestión del riesgo en el ámbito territorial comprende la gestión directa hacia las entidades, instituciones, organizaciones públicas y privadas involucradas con el desarrollo territorial del municipio.

Algunos referentes de los eventos naturales extremos como tormentas, huracanes tropicales, inundaciones, sequías, heladas, granizadas, terremotos, erupciones volcánicas, maremotos y aluviones deben tener herramientas claras que incluyan una metodología para la evaluación del impacto socioeconómico y ambiental de los desastres. Por ejemplo, en el caso de inversiones y localización de viviendas y su infraestructura complementaria, el Estado colombiano sigue siendo el ente responsable ante la sociedad para proveer y garantizar infraestructura adecuada en un hábitat en armonía; por lo tanto, no se 
debe construir en áreas con alta vulnerabilidad y se debe reforzar y asegurar la aplicación de las normas sobre asentamientos humanos y códigos de construcción más estrictos ligados a las condiciones de los riesgos.

Algunos casos locales han obligado a la articulación de los Planes de Desarrollo a inversiones territoriales por ajustes de los POT, como el terremoto de Armenia (Quindío) en el año 1999, deslizamiento del cerro Pan de Azúcar en el barrio Villatina (Medellín), el cual ocasionó la muerte de aproximadamente quinientos personas en el año 1987 y el más reciente, mayo de 2015, la avalancha que se generó a partir del desbordamiento de una quebrada cercana a la vereda Santa Margarita en el municipio de Salgar (Antioquia), identificado tanto en el POT 2012 como en el Plan de Desarrollo Local, que alertaron sobre una posible tragedia, pero no se tomaron las medidas respectivas. El Ideam cuestionó la construcción de viviendas a orillas de la quebrada.

Según el Plan de Desarrollo de Salgar, para evitar que sucediera este tipo de eventos había que llevar a cabo una serie de proyectos como obras de mitigación en zonas de alto riesgo, educar en la prevención de desastres, reubicar la escuela León de Greiff, hacer el análisis de riesgo para los corregimientos de La Cámara y Peñalisa y reubicar las diecinueve viviendas que estaban sobre la margen izquierda del río San Juan, entre otras (Avendaño, 2015).

Para el Gobierno nacional central, la experiencia de los sucesos climatológicos de 2010-2011 (efecto de La Niña) fue el detonante para el direccionamiento de algunas acciones en el desarrollo de políticas públicas, sobre todo en términos de inversión de los recursos financieros municipales por medio de las entidades departamentales. Para el caso de la ciudad de Bogotá, en asocio con el departamento de Cundinamarca, se ha trabajado en la solución de problemas comunes como las áreas compartidas a lo largo de la cuenca hidrográfica del río Bogotá. Se encontraron problemas derivados de la ocupación de rondas por asentamientos humanos de índole ilegal y legal, lo que lleva a tomar acciones en el planteamiento y ordenamiento alrededor del sistema hídrico como alternativa de desarrollo y crecimiento para municipios.

En términos de planificación, gestión y ordenamiento territorial, esto conduce a la articulación de los Decretos 1729 de 2002 y 1640 de 2012, referentes al manejo de cuencas hidrográficas, para garantizar la ejecución de programas y proyectos mediante los Planes de Desarrollo departamental y municipal; en cuanto a asociatividad territorial municipal, la Región Administrativa de Planificación Especial (RAPE) garantizará la ejecución de programas y proyectos más integrales, la cual garantizará la ejecución de planes de desarrollo en programas y proyectos regionales, según lo dispuesto en el Artículo 30, párrafo 3, de la Ley Orgánica de Ordenamiento Territorial 1454 de 2011. Bogotá, el departamento de Cundinamarca y los departamentos contiguos a este (Boyacá, Meta y Tolima) podrán asociarse en una RAPE.

En el marco normativo vigente se buscará la armonización territorial e institucional en la aplicabilidad de las Unidades de planificación y de gestión Territorial (UPGT) en asocio con los instrumentos de planificación conocidos PD-POT-Pomca-PLGR, donde se garantice la articulación al desarrollo ambiental, social y económico, la articulación con lo administrativo, y por supuesto se busque la disminución de la vulnerabilidad y el riesgo para futuros eventos asociados al agua, para ello, también la Ley Orgánica de Ordenamiento Territorial (LOOT 1454 de 2011) aporta una buena gama de opciones de armonización administrativas, que determinarían futuras asociaciones territoriales con el fin de compartir decisiones conjuntas en la solución de conflictos ambientales, entre ellas la puesta en marcha de las RAP, Regiones Administrativas de Planeación (Villegas, 2014). 


\section{Reformas institucionales}

\section{de impacto territorial y regional}

A continuación se presenta el esquema organizativo en la conformación y utilización de Unidades de Planificación y de Gestión Territorial (UPGT), situación que fortalece a la institución asociada en el manejo de la gestión del riesgo y su impacto en el territorio. Sigue siendo recurrente el debate sobre la articulación entre municipios y departamentos y su estructura administrativa para establecer las competencias en el momento del evento con respecto a las entidades de las Corporaciones Autónomas Regionales, las cuales pueden estar inscritas a territorios más amplios para fortalecer la integralidad de los asociados en términos regionales. $\mathrm{Al}$ tener una visión más completa del territorio, se asigna a municipios y departamentos de mayor presencia o fortaleza administrativa la sede del Comité de Integración Territorial (CIT), que son cuerpos colegiados en los que las autoridades competentes concertarán lo referente a la implementación de los Planes de Ordenamiento Territorial. Asimismo, serán escenarios de participación comunitaria e institucional, de acuerdo con el territorio conformado por las UGPT (Congreso de la República, 2000a).

En lo referente a la jurisdicción político-administrativa de las autoridades ambientales regionales, conocidas como CAR, no responde a criterios de manejo ambiental, sino que en la mayoría de los casos correspondió a criterios político-administrativo entre departamentos y municipios, lo que fraccionó el territorio ambiental en la toma de decisiones. Para una organización del país en cuanto al aspecto ambiental, el Plan Nacional de Desarrollo 2010-2014 reconoce a la estructura ecológica principal como instancia de planificación ambiental territorial, en la que se hace viable la propuesta de las UPGT de las instituciones que forman parte del SINA y que serían objeto de su armonización administrativa y territorial.

Las instituciones del Estado, como el Ministerio de Ambiente y Desarrollo Sostenible y sus divisiones administrativas, formarán parte de los Comités de Integración Territorial en el momento en el que se necesiten y estarán a su cargo la participación relacionada con la gestión del riesgo y la nueva Subdirección de Coordinación del Sistema Nacional Ambiental, lo cual parece fortalecer notablemente el grupo SINA. Esto deja entrever una relación mucho más activa entre entidades centrales con las autoridades departamentales y municipales. En temas regionales, el Gobierno nacional y la CAR, como entidad ambiental regional, proyectarán la coordinación en cada uno de los CIT que se establezcan.

Con esta proposición se busca hacer coincidir el diagnóstico ambiental de los departamentos con la caracterización ambiental de las CAR y los municipios; así se unificarán esfuerzos en términos de recursos económicos entre las partes y se podrá trabajar en los planes diseñados por los municipios, con su jurisdicción político administrativa vigente.

En la Figura 1 se determinan los "nuevos" alcances del SINA propuestos por el Gobierno nacional 2012. En cada categoría se relacionan las entidades o los actores que formarían parte de su soporte técnico y científico en la evaluación de la gestión del riesgo en la incorporación de los instrumentos de planificación y gestión territorial para el planeamiento del territorio. En cada bloque se determinaron sus respectivos alcances: en el primero, el SINA social, se asocian los Ecofondos, las ONG, organizaciones sociales y gremios sectoriales. El Consejo Nacional Ambiental (CNA) lo integrarán el MADS, el MVDT, los institutos de investigación, las Corporaciones Autónomas Regionales y de Desarrollo Sostenible 
y el subsistema de investigación ambiental, las unidades ambientales urbanas y la Unidad Administrativa Especial del Sistema de Parques Nacionales Naturales y el DNP.

Con esta nueva estructura del SINA, organizada en cuatro unidades, se espera que su funcionalidad demuestre mayor protagonismo entre las instituciones que la conforman en el caso de actuaciones futuras que tengan que ver con la presencia del Estado en la atención y gestión del riesgo.

En relación con la planificación y gestión territorial, las instituciones están determinadas por un régimen específico, que define un marco de maniobra en la intervención en el territorio, según lo establezca la ley. Esto no es ajeno a la superposición territorial de obligaciones al momento de definir intervenciones presupuestales, en la medida en que los Planes de Desarrollo, como instrumentos de planificación y de gestión territorial, determinan su intervención específica en presupuestos de inversión, su interrelación o complementariedad con los Planes de Ordenamiento Territorial, fundamental por la localización de la infraestructura.

Por lo tanto, la zonificación ambiental mediante la estructura ecológica principal muestra las áreas que deben estar comprometidas en las actividades económicas y ambientales, con los sectores y actores en el territorio, para que sean conservadas o desarrolladas según el régimen de usos del suelo establecidos por los POT y los Pomca que, como herramientas de gestión territorial, definen el ordenamiento del territorio. Las Corporaciones Autónomas Regionales serán

Figura 1.

Organigrama institucional del Sistema de Información Nacional Ambiental (SINA)

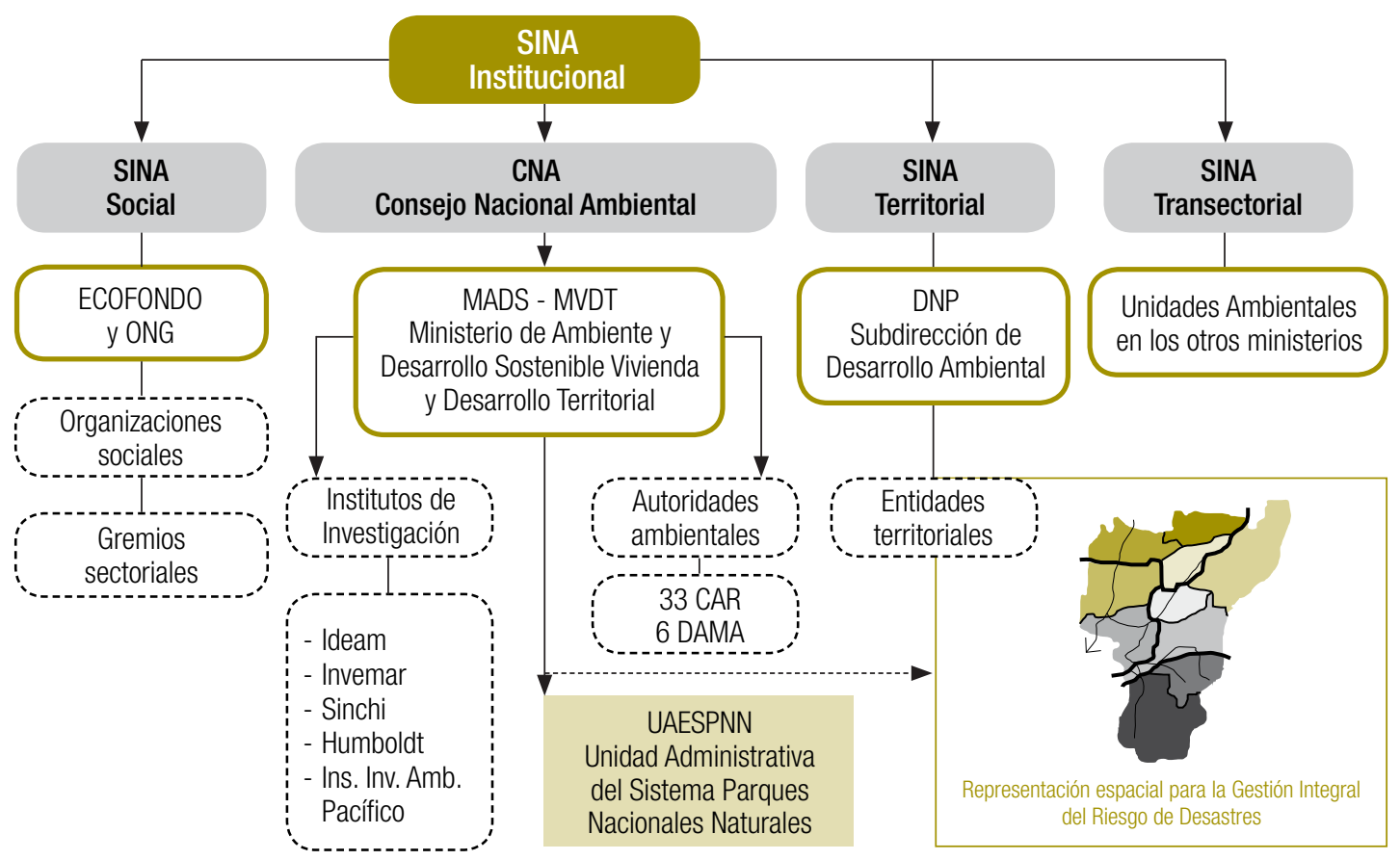

Fuente: elaboración propia 
las veedoras del cumplimiento en el desarrollo y crecimiento de los territorios y tendrán en cuenta cuáles áreas de ronda en el recurso hídrico no podrán ser ocupadas ni destinadas a disposiciones finales de residuos sólidos como vertimientos de aguas servidas; todo ello se hará en colaboración con entidades municipales. Para la integración de los POT, en el ańo 2000 se aprobó la Ley 614, cuyo objetivo es establecer mecanismos de integración, coordinación y armonización de las entidades competentes para la implementación de los planes de ordenamiento territorial. Por medio de los Comités de Integración Territorial (CIT) tendrán mayor participación, al tener como instrumento de planificación la gestión del recurso hídrico mediante la zonificación ambiental territorial y la estructura ecológica principal, diseñada desde la cuenca hidragráfica para una mayor armonización territorial.
Asimismo, los CIT podrán garantizar la presencia de presupuestos integrales con inversiones similares entre entidades municipales y departamentales en el manejo de inversiones en prevención y atención de desastres. Según la Ley 1523 del 24 de abril de 2012, el Consejo Nacional para la Gestión del Riesgo de Desastres y la Unidad para la Gestión del Riesgo de Desastres se coordinan por tres comités a saber: el Comité Nacional para el conocimiento de riesgos de desastres; el Comité nacional para la reducción del riesgo de desastres y el Comité nacional para el manejo de desastres, direccionados por la Junta Directiva del Fondo Nacional de GRD, en donde gobernadores y alcaldes fortalecidos por los Consejos Territoriales ajustan las políticas públicas (Figura 2). Lo anterior estará inmerso en los CIT que se establezcan en toda el área conformada por la armonización territorial.

Figura 2.

Estructura organizacional del Sistema nacional para la gestión del riesgo de desastres (SNGRD)

Presidencia

de la República

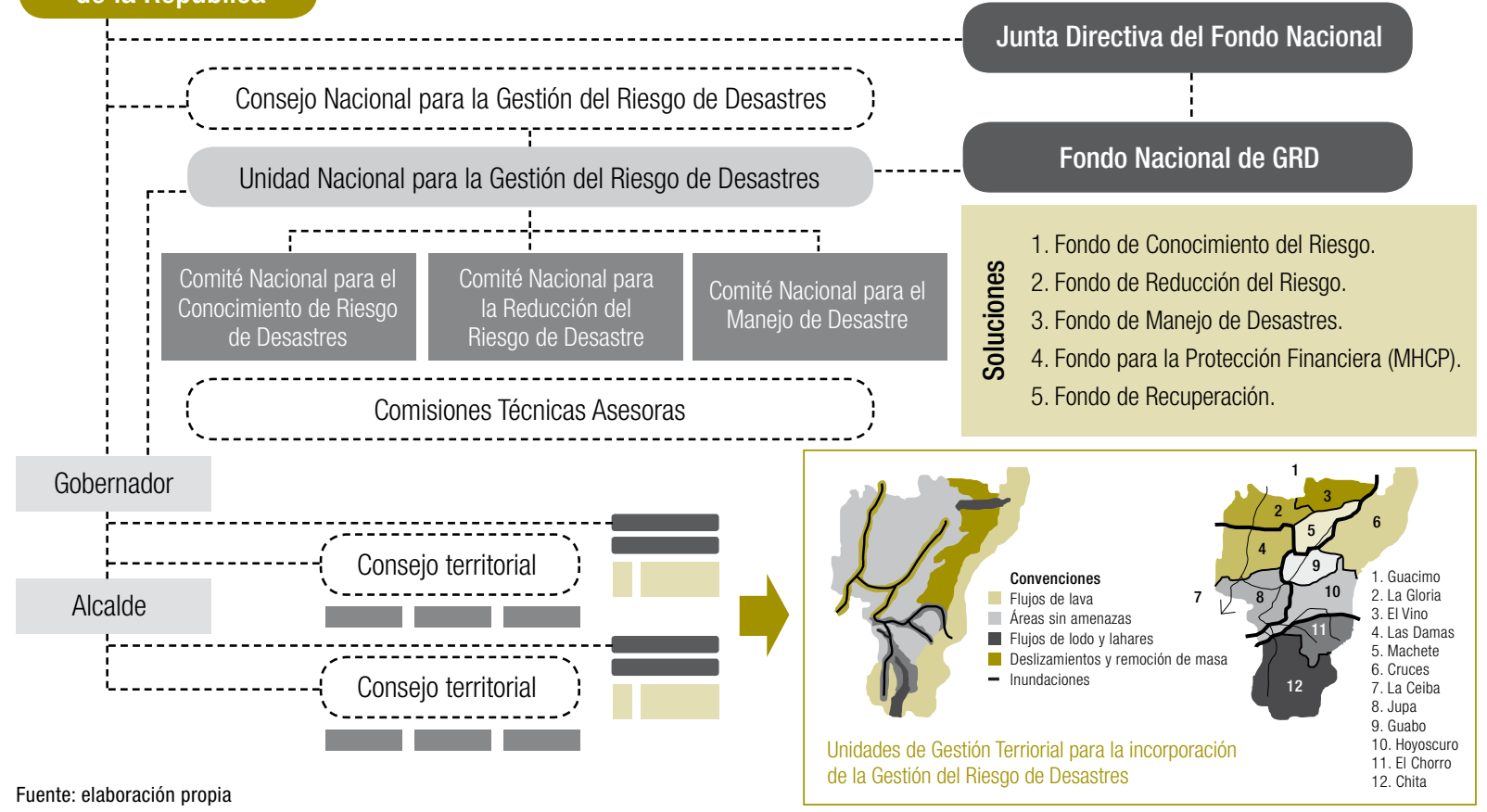




\section{Articulación territorial e institucional en lo normativo en el caso de los PD, POT y Pomca}

Como se mostró en los dos pasos anteriores, la incorporación del riesgo permitirá definir medidas estructurales para la prevención y mitigación orientadas a la reducción del riesgo existente y evitar la generación de nuevos riesgos en los municipios. Un Comité de Integración Territorial (CIT) será la entidad territorial que incorpore la gestión del riesgo dentro de su proceso de planificación territorial. Los CIT serán cuerpos colegiados en los cuales las autoridades competentes concertarán lo referente a la implementación de los Planes de Ordenamiento Territorial y a la presentación de la visión estratégica de desarrollo futuro del área de influencia territorial, cuyo eje central será la gestión del riesgo en cinco escenarios identificados para su trabajo interdisciplinario, a saber:

1. Apropiado conocimiento en relación con los fenómenos potencialmente peligrosos y las áreas de tales fenómenos.

2. Zonificación de la aptitud para el uso del suelo, que considere fenómenos potencialmente peligrosos para áreas no ocupadas.

3. Zonificación de las áreas ya ocupadas que presentan susceptibilidades de sufrir daños por deslizamientos e inundaciones.

4. Reglamentación para evitar las ocupaciones que generarían riesgos y por consiguiente desastres (suelo de protección o de uso con restricciones ambientales).

5. Reglamentación para priorizar las acciones que se deben ejecutar para reducir el riesgo hasta un grado aceptable, por ejemplo, la reubicación de asentamientos e inmuebles de desarrollo económico y social vistos en su totalidad en cada uno de los POT y Pomca sobre el suelo urbano, de expansión urbana y de protección ubicados en áreas urbanas; en tal sentido, orientar y planear su ocupación, prevenir y mitigar actividades o eventos naturales que puedan incidir de forma imprevista sobre las actividades y las personas que se encuentren en dichos suelos. Sin embargo, en la actuación del riesgo, cada uno de los municipios que integren el área dispone de herramientas de gestión y financiación, que permiten sus actuaciones en momentos y circunstancias diferentes de crisis: la asistencia tendrá incidencia en la aplicación de las siguientes herramientas de gestión y de planificación.

a. Herramientas para sectores específicos que requieren un proceso de planificación en detalle para concretar lo propuesto en el POT, planes parciales y macroproyectos urbanos (actuaciones urbanas integrales).

b. Herramientas para garantizar el reparto equitativo de cargas y beneficios: unidades de actuación urbanística, compensaciones, transferencia de derechos.

c. Herramientas para la adquisición de inmuebles: enajenación voluntaria, enajenación forzosa, expropiación administrativa, expropiación judicial, derecho de preferencia.

d. Herramientas para dinamizar el desarrollo de sectores inactivos o deteriorados: declaratoria de desarrollo prioritario, derecho de preferencia.

e. Herramientas que complementan la financiación del desarrollo territorial: participación en plusvalías, valorización y bonos de reforma urbana.

La armonización de estas herramientas debe incluirse en la ordenación territorial en todo el territorio asociado. 
En síntesis, la incorporación y el reconocimiento de la Estructura Ecológica Principal (EEP) y la Zonificación Ambiental (ZA) como herramientas para la ordenación del territorio, los Planes de Desarrollo (PD), los Planes de Ordenamiento Territorial (POT) y los Planes de Manejo Integral de Cuencas Hidrográficas (Pomca) deberán resaltar los avances en la incorporación de la dimensión ambiental y priorizar, entre los principales retos, el territorio sostenible y la identificación y caracterización de los servicios ecosistémicos de los que dependerán las nuevas generaciones, posiciones que serán ordenadas por el Plan Nacional de Desarrollo y serán tenidas en cuenta por los territorios asociados que, en muchos casos, no han sido consultados por las entidades municipales. Lo anterior dará un paso a una nueva generación de instrumentos de planificación y de gestión territorial denominados PD, POT, Pomca de segunda generación.

Las Figuras 3 y 4 muestran la relación entre los planes mencionados y la propuesta esquemática para la armonización, pero por la extensa normatividad local también serán determinantes en el momento de relacionar las visiones gubernamentales de los municipios que han desarrollado. Como parte del sistema de planificación regional,

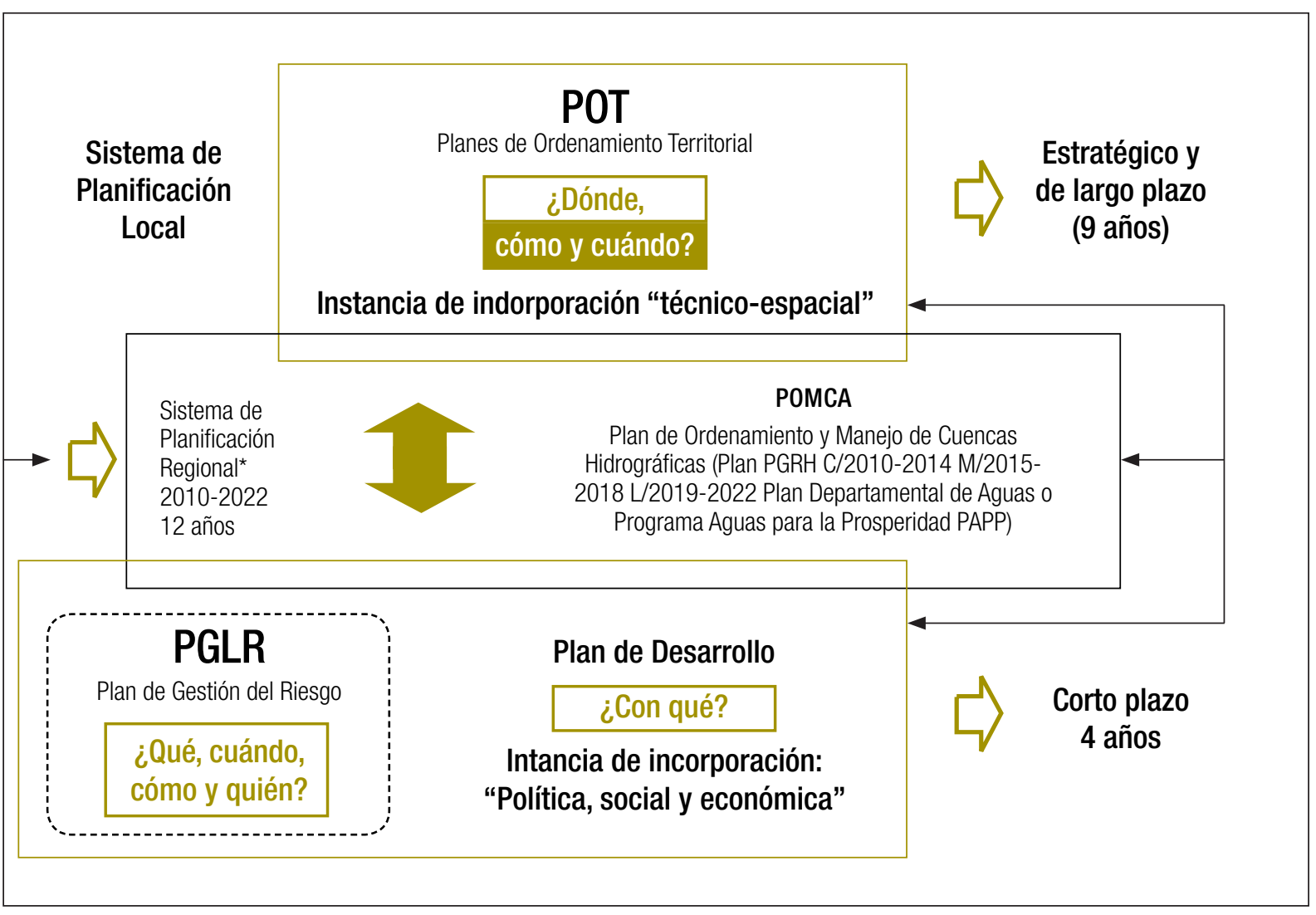


se viene incorporando el manejo de los Planes de Gestión del Recurso Hídrico (PGRH) articulados desde el Plan Departamental de Aguas, estructurado hacia la formulación de los planes del recurso hídrico $(\mathrm{PORH})$, según la última guía para su formación e implementación de 2014 promulgada por el Ministerio de Ambiente y Desarrollo Sostenible.

La evaluación de la gestión del riesgo compromete varios instrumentos de planificación y de gestión territorial, como se identifica en la Figura 3, los cuales estarán en constante relación con las instancias territoriales. Para ello, se proponen tres pasos: a. La división institucional territorial.

b. El mapa de riesgos y amenazas.

c. El escenario propositivo en la armonización y la gestión territorial en los planteamientos de las Unidades de Planificación y Gestión Territorial (UPGT).

La Figura 4 muestra la importancia de tener en cuenta la división político-administrativa existente y el mapa de riesgos y amenazas, para determinar la zonificación de las unidades territoriales de gestión para la armonización de programas y proyectos ejecutables mediante los distintos de desarrollo planes de ordenamiento y Pomca. Por

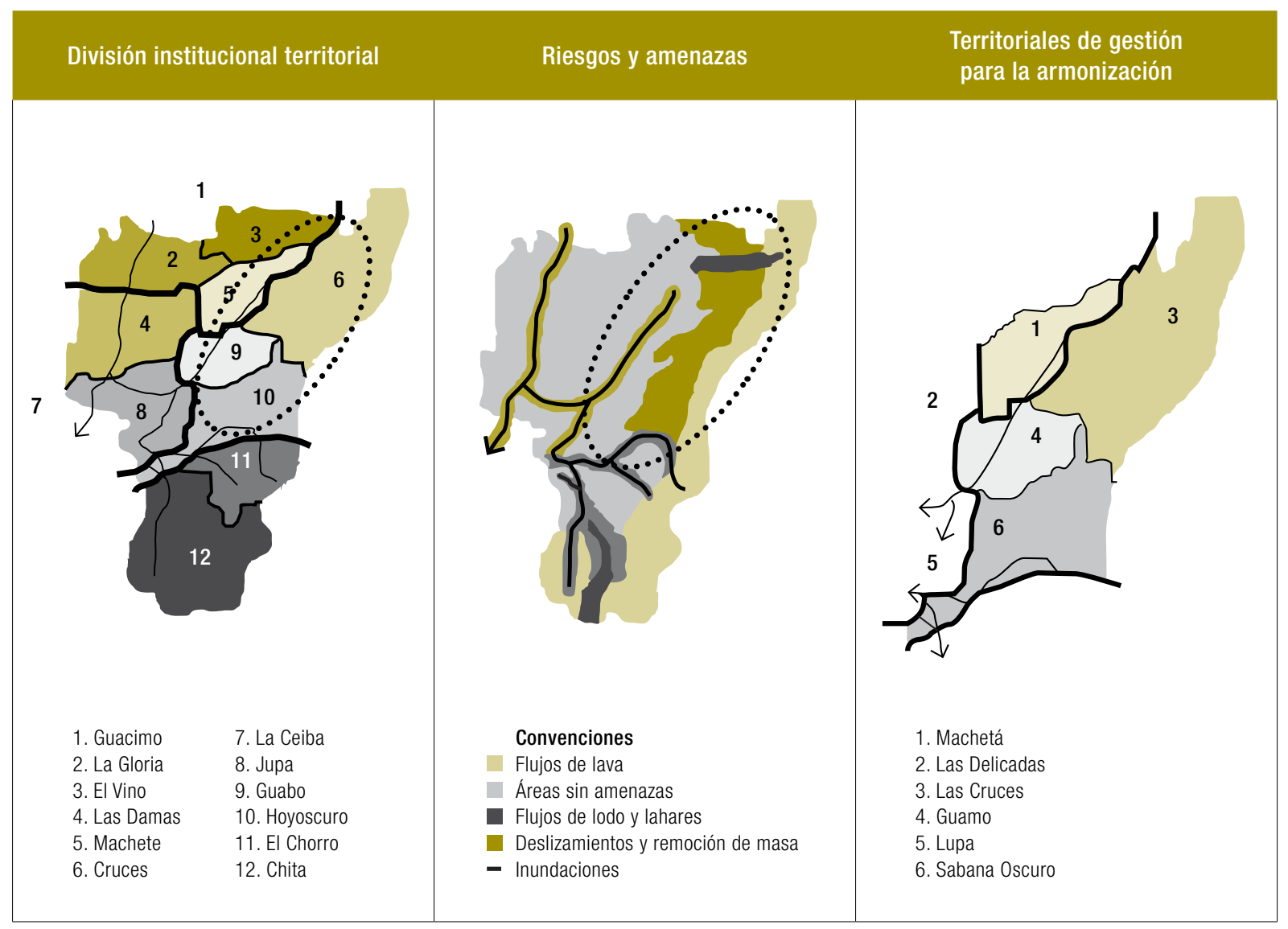


tal motivo, es fundamental identificar algunos instrumentos de planificación y de gestión territorial como el PD, el POT y los Pomca, en particular este último, porque proporciona las bases o condiciones geográficas (ambientales) para determinar los grados de asociatividad con municipios y departamentos; esto constituye un medio de articulación para llevar a cabo el desarrollo de políticas públicas entre ellas.

Con ello, no solo se quiere obtener más provecho de lo que ya existe, sino perfeccionar los mecanismos de aplicación de programas, con el ánimo de que más poblaciones rurales se vean beneficiadas por la llegada de nuevos proyectos de infraestructura coordinados entre los municipios asociados y, por supuesto, en armonía con las posibilidades del desarrollo del recurso hídrico, coberturas de agua potable y el desmonte de los Planes Departamentales de Aguas (PDA), para dar continuidad a la nueva vida al programa Agua para la Prosperidad (Departamento de Planeación Nacional, 2010).

La Figura 5 involucra los distintos instrumentos de gestión institucional actuantes en el territorio dentro de cada uno de los municipios, con el fin de proyectar estrategias sostenibles en

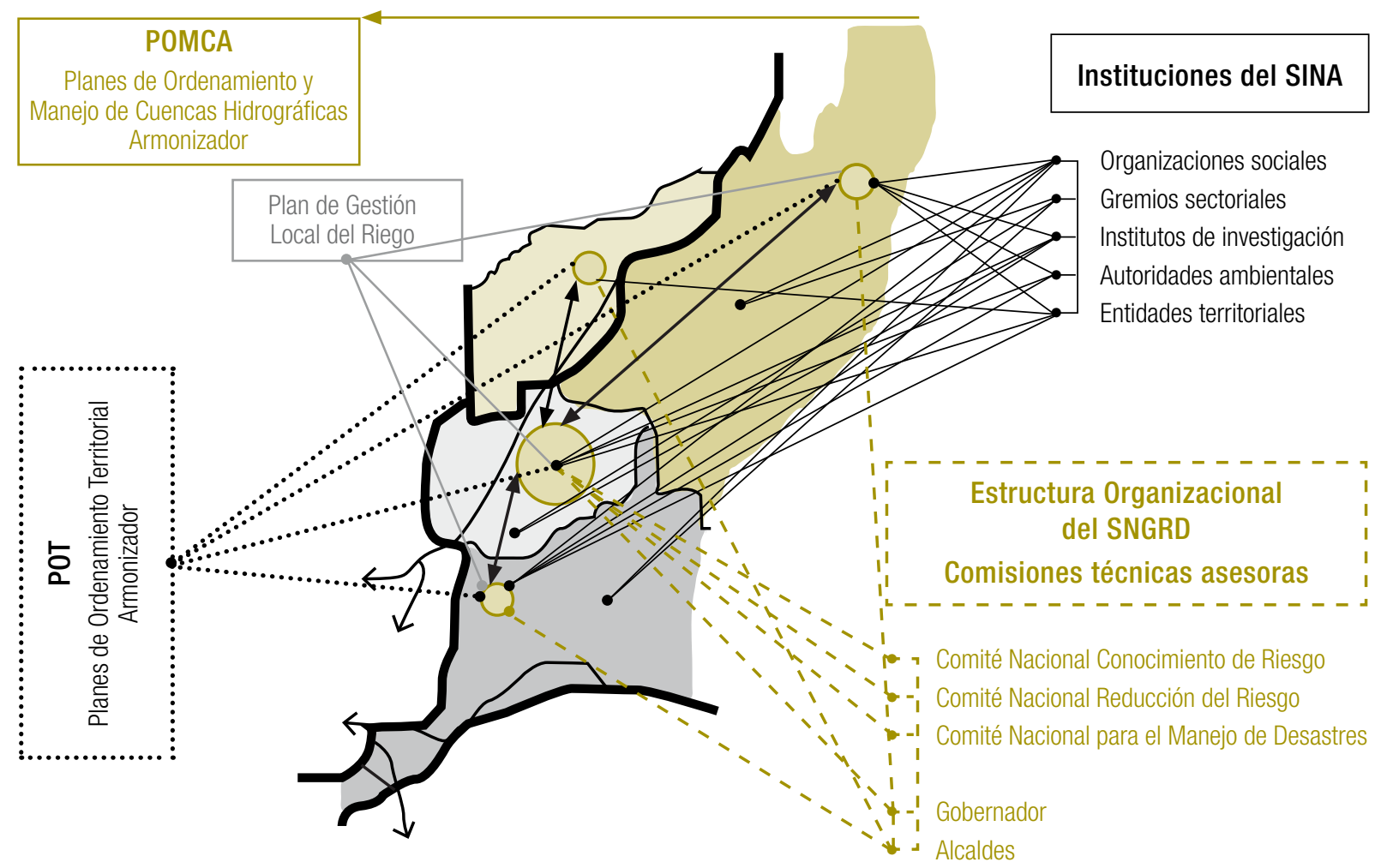


conjunto para la gestión del riesgo, de acuerdo con la ocupación y participación de los distintos actores en el territorio. Como lo hemos venido planteando, la armonización de los instrumentos, su utilidad y su determinación se hace bajo el objetivo estratégico de las Unidades de Planificación y Gestión Territorial (UPGT)

De manera específica, las UPGT gestionarán institucionalmente la actuación de organizaciones y sus vínculos interinstitucionales; además, identificarán las necesidades territoriales que requieran mayor atención para su intervención inmediata, la cogestión entre entidades y el fortalecimiento de las mismas relaciones en su conjunto lo cual robustecerá la participación de la sociedad civil, puesto que estará atenta a la inclusión en sus decisiones tanto en la inversión de programas y proyectos cuyo objetivo es la prevención de riesgos naturales.

La localización de recursos financieros por medio de las UPGT permitirá la planificación, en términos de gestión financiera, de la armonización de los territorios socios; así, se verán con claridad los esfuerzos en la ejecución de infraestructura y se prevendrán eventos que pongan en riesgo la vida en algunos asentamientos poblacionales que no pueden ser reubicados, pero sí pueden ser intervenidos. Esto disminuirá la amenaza y la vulnerabilidad ante un evento, ya sea por deslizamiento o inundación. Las competencias territoriales deben quedar claras en la participación de cada uno de los actores directamente involucrados e identificados en la propuesta de las Unidades de Planificación y de Gestión Territorial, base en el fortalecimiento de la gestión territorial para prevenir el crecimiento poblacional en áreas no aptas para su localización y mejorar el desarrollo social de la población y su calidad de vida, al disminuir su grado de vulnerabilidad ante las amenazas.

\section{Conclusiones}

En este artículo emergen varios elementos con los cuales se puede establecer la síntesis de políticas, instituciones e instrumentos para la articulación del SNGRD, la cual puede servir como base de un trabajo orientado a mejorar las condiciones favorables de la vinculación de la gestión territorial al manejo de la gestión de desastres. Del análisis y estudio se destacan algunos elementos para tal interrelación, como el Sistema de Información Ambiental (SINA) desde la nueva estructura propuesta por el Estado. En ella se determinaron las características más importantes para su funcionamiento transversal, con el planteamiento y reconocimiento del SINA social, en el que están representados la organización Ecofondos, las ONG, las organizaciones sociales y los gremios sectoriales; paralelamente, se articula el Consejo Nacional Ambiental, liderado por la estructura del nuevo Ministerio de Ambiente y Desarrollo Sostenible y el Ministerio de Vivienda y Desarrollo Territorial, en el que se integran los institutos de investigación y autoridades ambientales, el SINA territorial —liderado por el DNP - y las entidades territoriales municipales.

La inclusión de las acciones administrativas en el tema de gestión del riesgo de desastres se hará desde la Presidencia de la República y los gobernadores y alcaldes estarán coordinados por el Consejo Nacional de la Unidad Nacional para la Gestión del Riesgo de Desastres, direccionados desde los tres comités: Conocimiento, Reducción y Manejo del Riesgo de Desastres.

Esta es la nueva estructura del Sistema Nacional para la Gestión del Riesgo de Desastres, vigente para afrontar los impactos del cambio climático y el calentamiento. 
El esquema propuesto se confronta con el desarrollo de acciones y estrategias en el territorio, por medio de los instrumentos y las herramientas de planificación y de gestión territorial que están directamente relacionados con la continuidad de inversiones en algunos programas y proyectos de los Planes de Desarrollo (PD), verificados en los municipios.

Los Planes de Ordenamiento Territorial (POT) y los Planes de ordenamiento y manejo de cuencas hidrográficas (Pomca) estarán en un inminente riesgo para su ejecución, si no se incorporan algunas decisiones de políticas públicas nacionales de intervención municipal que, por su estructura de inversión estatal, por ejemplo, el proyecto minero, son consideradas por el Estado central como de mayor jerarquía; por ello, no serán acogidas por los POT y los Pomca municipales, lo que pone en desventaja la participación de sectores de la sociedad civil, que deberá estar informada con anterioridad a la ejecución de macro o mega proyectos en su territorio. Además de los proyectos mineros, podemos identificar los hidroeléctricos que, por su dimensión, obligan a que los municipios involucren la variabilidad de la gestión del riesgo en sus Planes de Desarrollo, Planes de Ordenamiento y manejo de cuencas.

En términos generales, las recomendaciones pueden estar estructuradas sobre dos bases: la primera, el aporte académico al debate de los mecanismos de regulación estatal acerca del desarrollo de las inversiones en el territorio en proyectos elegibles, cuyo impacto incide en la apropiación y modificación de nuestro patrimonio natural; segunda, la institucional, determinante de la inversión pública en el desarrollo territorial por medio de la inclusión de políticas públicas que inciden directamente en el fortalecimiento de las Administraciones territoriales, las cuales deben ver reflejadas sus inversiones en términos globales y regionales.

El proyecto de investigación es complementario a deliberaciones en el manejo de directrices, conceptos y metodologías, que deben incorporarse en la gestión territorial, directamente desde la posición de las Administraciones que tienen a su cargo el desarrollo y la inversión pública, para fortalecer la inversión privada e incluir a la comunidad en general en la construcción del territorio.

Algunas relaciones identificadas en este artículo pueden ser desarrolladas con la ayuda de los métodos de gestión de unidades territoriales, cuya finalidad es fortalecer la armonización territorial en el marco de la cogestión entre las asociaciones público-privadas y la participación de la sociedad civil, de acuerdo con los grados de prevalencia considerados como determinantes para agrupar o asociar entidades territoriales, con el propósito de robustecer los lazos de gestión entre distintos actores, sectores y gremios que definen las políticas públicas, con el fin de planificar y gestionar el desarrollo territorial.

\section{Bibliografía}

Avendaño, M. L. (2015). Salgar llora su mayor catástrofe. El Espectador. Recuperado de http:// www.elespectador.com/noticias/nacional/salgar-llora-su-mayor-catastrofe-articulo-561461

Congreso de la República (1993). Ley 99 de 1993, "Por la cual se crea el Ministerio del Medio Ambiente, se reordena el sector público encargado de la gestión y conservación del medio ambiente y los recursos naturales renovables, se organiza el Sistema Nacional Ambiental SINA y se dictan otras disposiciones". Bogotá: Diario Oficial No. 41146, diciembre 22 de 1993. 
Congreso de la República (1997). Ley 388 de 1997, "Por la cual se modifica la Ley 9 de 1989 y la Ley 2 de 1991 y se dictan otras disposiciones”. Bogotá: Diario Oficial No. 43.091, julio 18 de 1997.

Congreso de la República (2000a). Ley 1454 de 2011, "Por la cual se dictan normas orgánicas sobre ordenamiento territorial y se modifican otras disposiciones". Bogotá: Diario Oficial No. 48.115, junio 29 de 2011.

Congreso de la República (2000b). Ley 614 de 2000, "Por medio de la cual se adiciona la Ley 388 de 1997 y se crean los comités de integración territorial para la adopción de los planes de ordenamiento territorial". Bogotá: Diario Oficial No. 44.168, septiembre 20 de 2000.

Congreso de la República (2012). Ley 1523 de 2012, "Por la cual se adopta la política nacional de gestión del riesgo de desastres y se establece el Sistema Nacional de Gestión del Riesgo de Desastres y se dictan otras disposiciones". Bogotá: Diario Oficial No. 48.411, abril 24 de 2012.

Consejo Nacional de Política Económica y Social, Departamento Nacional de Planeación (2007). Documento Conpes No 3463, Planes departamentales de agua y saneamiento para el manejo empresarial de los servicios de acueducto, alcantarillado y aseo. Bogotá: Autor.

Consejo Nacional de Política Económica y Social, Departamento Nacional de Planeación (2011). Documento Conpes No 3700, Estrategia institucional para la articulación de politicas y acciones en materia de cambio climático en Colombia. Bogotá: Autor.

Departamento Nacional de Planeación [DNP] (2010). Plan Nacional de Desarrollo 2010-2014. Recuperado de https://www.dnp.gov.co/Plan-
Nacional-de-Desarrollo/PND-2010-2014/Paginas/Plan-Nacional-De-2010-2014.aspx

Ideam (2011). Aportes del Ideam para la definición y aplicación de la Estructura Ecológica Nacional. Bogotá: Autor.

Lozano Vila, A. (2011). La institucionalidad ambiental en Colombia frente al cambio climático y la ola invernal. Bogotá: Konrad Adenauer Stiftung.

Ministerio de Ambiente, Vivienda y Desarrollo Sostenible (2012). Estructura Organizacional del Nuevo SINA. Bogotá: Autor.

Ministerio de Ambiente, Vivienda y Desarrollo Territorial (2005). Guía metodológica 1. Incorporación de la prevenvción y reducción de riesgos en los procesos de ordenamiento territorial. Bogotá: Autor.

Ministerio de Ambiente, Vivienda y Desarrollo Territorial (2010). Política nacional para la gestión integral del recurso hidrico. Bogotá: Autor.

Presidencia de la República (2002). Decreto 1729 de 2002, "Por el cual se reglamenta la Parte XIII, Título 2, Capítulo III del Decreto-ley 2811 de 1974 sobre cuencas hidrográficas, parcialmente el numeral 12 del Artículo 5 de la Ley 99 de 1993 y se dictan otras disposiciones". Bogotá: Diario Oficial No. 44.893, agosto 7 de 2002.

Presidencia de la República (2007). Decreto 3600 de 2007, "Por el cual se reglamentan las disposiciones de las Leyes 99 de 1993 y 388 de 1997 relativas a las determinantes de ordenamiento del suelo rural y al desarrollo de actuaciones urbanísticas de parcelación y edificación en este tipo de suelo y se dictan otras disposiciones". Bogotá: Diario Oficial No. 46.757, septiembre 20 de 2007.

Presidencia de la República (2011). Decreto 4147 de 2011, "Por el cual se crea la Unidad Nacional para la Gestión del Riesgo y de Desastres, se 
establece su objeto y su estructura”. Bogotá: Diario Oficial No. 48.242, noviembre 3 de 2011.

Presidencia de la República (2012). Decreto 1640 de 2012, "Por medio del cual se reglamentan los instrumentos para la planificación, ordenación y manejo de las cuencas hidrográficas y acuíferos y se dictan otras disposiciones". Bogotá: Diario Oficial No. 48.510, agosto 2 de 2012.

Procuraduría General de la Nación (2007). Seguimiento a la ordenación de cuencas hidrográficas en Colombia. Informe preventivo. Bogotá: Autor.

Villegas, E. (2014). Las unidades de planificación y gestión territorial como directriz para la zonificación urbana. El Ágora USB, 14(2), 551-581. 